\title{
An Improved Image Fusion Scheme Based on Markov Random Fields with Image Enhancement Scheme
}

\author{
Nalla Aravind Babu \\ Department of ECE \\ Vardhaman College of Engineering \\ Hyderabad, India
}

\author{
M. Veeraswamy \\ Department of ECE \\ Vardhaman College of engineering \\ Hyderabad, India
}

\begin{abstract}
Image fusion is an important field in many image processing and analysis tasks in which fusion image data are acquired from multiple sources. In this paper, we investigate the Image fusion of remote sensing images which are highly corrupted by salt and pepper noise. In our paper we propose an image fusion technique based Markov Random Field (MRF). MRF models are powerful tools to analyze image characteristics accurately and have been successfully applied to a large number of image processing applications like image segmentation, image restoration and enhancement, etc.,. To de-noise the corrupted image we propose a Decision based algorithm (DBA). DBA is a recent powerful algorithm to remove high-density Salt and Pepper noise using sheer sorting method is proposed. Previously many techniques have been proposed to image fusion. In this paper experimental results are shown our proposed Image fusion algorithm gives better performance than previous techniques.
\end{abstract}

Keywords: Markov Random Field, image fusion, image segmentation, image restoration and enhancement, Decision based algorithm.

\section{INTRODUCTION}

The applications were image data should be gathered (or collected) from many input images as source images, in such applications image fusion plays an important role. The source images contain about some information of the original scene but individually they cannot represent full scene perfectly in order to gather the original scene from the source images image fusion is used. The input images for fusion can be capture at different time and/or by an utilizing various sensors, because of this source images individually cannot represent full scene [1]. Thus we perform image fusion in order to get an accurate view at the scene, that's why it is more applicable in many image processing applications [2]. In applications like satellite image sensing image fusion is utilize to improve the resolution of the images [3], [4]. Here, we propose fusion scheme for source images having same resolution. The multispectral fusion scheme consists of a range of bands so as to improve spectral resolution [4]. We use several bands improving spectral resolution.

The available schemes of image fusion can be broadly classified into three categories: feature level, decision level and pixel level. In our paper we are using pixel level type of image fusion; so many techniques have been proposed of image fusion for various applications. In general there are two steps involved in pixel level image fusion.

a) We have to estimate whether given source image will contribute to the final fused image or not. For each and every pixel.

b) By using all the source images the intensity of the fused image can be formed.

The true previous techniques of fusion are by averaging pixel intensities of input images or by taking maximum pixel intensity out of total available source images pixel values. Gaussian noise can be effectively removed in case of averaging scheme and also it increases SNR value but fails to maintain proper contrast.
Recently, a name approach has been introduced i.e. MD-based scheme (multi scale-decomposition) and it shows better results for various applications of image processing. It consists of following three steps.

a) The input images are decomposed into $\mathrm{n}$ levels by using wavelet transform.

b) At, each and every level of source images the fusion approach is applied.

c) Convert the image spatial domain by applying inverse wavelet transform, in order to synthesize the resultant image.

This MD-based approach attains good performance and increases computational complexity became use of wavelet transform, so depending on application requirement one can select or skip the transformation step. The pixel level fusion approach may introduce distortion ine 2 the resultant fused image, because of the drawback of pixel level fusion that the decision on a specific input image or not, is difficult.

This noteworthy to consider spatial co-relation while we are doing fusion and the users such model will increase the performance, one of the way to use this spatial co-relation is by the use of window based or region based method [5][6][8] .

Here in our paper we introduce MRF(Markov Random Field) as it has been used in image processing applications such as change detection[10], image de-noising [9][12], edge detection [11] and image restoration [13][14].

MRF can also be used for pixel level image fusion. The first algorithm focus on decision making as an MRF and the next algorithm both on decision making and exact image as an MRF and the contrast can be improved by finding the upper and lower band intensity values for the resultant image. Our scheme can be tested in the presence of salt and pepper noise and impulse noise as well and it shows better performance.

\section{PROBLEMM FORMULATION}

The main aim of imager fusion is to predict the specified scene, by considering that every source image has good view 

2012

of small part of scene [1]. Consider there are source images each can be represented as,

$$
y i(r)=H i x(r)+w i(r), \quad i=1, \ldots \ldots, N
$$

Where $r$ represents spatial coordinates, $y_{\mathrm{i}}(\mathrm{r})$ is the brightness or intensity of $i$ th image, $x(\mathrm{r})$ is the intensity of original scene or source scene at $\mathrm{r}$ which has to be predicted $w_{\mathrm{i}}(\mathrm{r})$, we presents noise and sensor selectivity coefficient is represented by $\mathrm{H}_{\mathrm{i}}(\mathrm{r})$. The above equation (1) represents a relationship between actual scene and input image; in practice this model has some drawbacks. The images captured from various sensor gives different aspects of original scene in this case this scheme is an approximation.

The fusion problem necessarily include the prediction of $x$ and $\mathrm{H}_{\mathrm{i}}$. Previous algorithms may also be represented by above model. In case of averaging $\mathrm{H}_{\mathrm{i}}=1$ for any $i$ and for maximizing case $\mathrm{H}_{\mathrm{i}}=1$ if $i=\max _{i}\left\{y_{i}\right\} ; \mathrm{H}_{i}=0$ otherwise. If $\mathrm{H}_{i}$ is known the gray scale values of fused image will be calculated by least square scheme [15] as,

$$
\vec{x}=\left(H^{T} H\right)^{-1} H^{T} Y(2)
$$

Where $\mathrm{H}$ is a vector having values $\left[\mathrm{H}_{1}, \mathrm{H}_{2}, \ldots, \mathrm{H}_{\mathrm{N}}\right]^{\mathrm{T}}$ and $\mathrm{Y}$ is a vector $\left[y_{1}, \mathrm{y}_{2}, \ldots \ldots, \mathrm{y}_{\mathrm{N}}\right]^{\mathrm{T}}$. in general $\mathrm{H}$ will be available without any information about $\mathrm{H}$ to predict the values of $\mathrm{H}$. the LS algorithm says that $\mathrm{H}$ will take the values which generates highest energy i.e.

$$
\begin{aligned}
\vec{E} & \left.=\min _{H}(Y-H X)^{T}(Y-H X)\right) \\
& ={ }_{H}^{\min }\left\{Y^{T} Y-\left(Y^{T} H\right)\left(H^{T} H\right)^{-1}\left(H^{T} Y\right)\right\} \\
& \left.=\max _{H} f\left(Y^{T} H\right)\left(H^{T} H\right)^{-1}\left(H^{T} Y\right)\right\}
\end{aligned}
$$

$\mathrm{H}_{\mathrm{i}} \in\{0,1\}, \mathrm{H}$ has $2^{\mathrm{N}}$ possible values, now the values $x$ will be estimated by LS technique as

$$
\dot{x}=\left\langle\hat{A}^{2} \hat{H}\right)^{1} \hat{A}^{2} y(4)
$$

The above scheme is sensitive to noise as $\mathrm{H}$ as well as intensity of resultant image $x$ estimated pixel. The accuracy of estimation of $\mathrm{H}$ coefficients has much impact on resultant image accuracy. A simple way is to consider that the pixel values lie in a small window will be constant now choose coefficients which generates highest energy in that window [7]. The main aim of LS technique is to reduce the error $\|y-y\|^{2}$, a famous scheme for enhancing the predicted error of LS is by incorporation of prior knowledge of $\mathrm{H}$ or $x$ [16]. We presented to use an MRF model in order to predict the coefficients of $\mathrm{H}$. it is predicted to enhance the accuracy of $\mathrm{H}$ coefficients and resultant image quality.

\section{PROPOSED ALGORITHM}

The problem in image fusion is to predict the original scene. The estimation of $\mathrm{H}$ is performed first before we go for $x$, which will decide whether the previous work we predict the value of $\mathrm{H}$ by using maximizing, LS, Averaging and Windowing scheme. Here in this session we represent the two schemes based on MRF which will consider the spatial coordinates as well. Therefore, the intensity value in the resultant image will be decided by intensity of source images and also by neighboring pixels as well. The first scheme (MRF_H) with MRF models the coefficients only, but in the case of second algorithm coefficient as well as fused image will be modeled via MRF (MRF_HX) few notations.

- $\quad \mathrm{X}$ : total original scene;

- $\mathrm{H}_{\mathrm{i}}: i$ th source image coefficients;

- $\quad \mathrm{Y}_{\mathrm{i}}: i$ th source image intensity;

- $\mathrm{H}$ : source images coefficients, where $\mathrm{H}(\mathrm{r}, \mathrm{i})=\mathrm{H}_{\mathrm{i}}$;

- $\quad Y$ : source images intensities, where $Y(r, i)=Y_{i}$

A. Image fusion: $\mathrm{H}$ coefficients will be modeled using MRF.

This approach is motivated by the fact that an input images coefficient has spatial correlation. We proceed with $\mathrm{H}$ coefficients via MRF. Assume a sites in an image be represented by ' $S$ ' and $A \in\{0,1, \ldots, \mathrm{L}-1\}$. The phase space with [14] the marginal pdf $\mathrm{H}$ can be expressed as below (5) with normalization constant $\mathrm{Z}_{\mathrm{H}}$ is given below.

$$
\begin{gathered}
\mathrm{P}_{\mathrm{H}}(\mathrm{H})==\frac{1}{z_{H}} \exp \left[-\frac{1}{2} \sum_{c \in s} U_{c}(H)\right](5) \\
Z_{H}(H)=\sum_{H \in A} \exp \left[-\frac{1}{T} \sum_{\operatorname{cs} S} U_{c}(H)\right]
\end{gathered}
$$

The value can be express in (6) and the optimal value of $\mathrm{H}$ can be represented in (7). The steps involved in these algorithms are as follows,

1) First, find the initial estimate of $H$ and $x$ and decide initial parameters and a looping variable has to be initiated in looping variable.

2) For every iteration a new estimate of $\mathrm{H}$ will be obtained by Gibbs pdf and with Gibbs potential $\mathrm{E}(\mathrm{H})$.

3) Make obtaining the resultant fused image by using (4).

4) Reduce the value of looping variable and repeat above (2) and (3) steps till convergence.

B. Image fusion: $\mathrm{H}$ coefficients and $\mathrm{x}$ image will be modeled.

The resultant fused image also consists of properties of spatial coefficients. Therefore we assume that the resultant fused image will also followed Markov Random Field along with Gibbs potential $\mathrm{Vc}(\mathrm{X})$. Therefore X marginal pdf in [9].

$$
P_{X}(X)=\frac{1}{z_{X}} \exp \left[-\frac{1}{2} \sum_{c c s} v c(X)\right]
$$

Here $Z_{X}$ is normalization constant and can be written as (6) by this consideration the optimal $X$ given as,

$$
\left.R^{n+1}-\arg _{H} \max _{H}\left[P\left(x \mid x, Z^{n}\right)\right]\right)
$$

Therefore the $\hat{H}$ estimation will reduces to,

$$
\begin{aligned}
& \left.\qquad \hat{X}^{n+1}=\arg \int_{X}^{\max }(\Delta(X))\right] \\
& \text { Where, } \\
& \Delta(X)=\frac{1}{2 \sigma^{2}} \sum_{i} Y_{i}-A_{i}^{n} X^{\mathrm{T}}\left(Y_{i}-A_{i} X\right)+\sum_{n=s} V c(X)
\end{aligned}
$$


As compare to scheme proposed previously i.e. MRF_H in that by using LS algorithm we updated the fused image $X$. in this technique we use (10) to update the resultant image the algorithm is as follows.

1) As a first step de-noise the input images.

2) Start with an initial estimate of $H$ and $X$. Estimate the initial parameters and set the initial variable.

3) For every iteration new $\mathrm{H}$ vale will be obtain on the basis of its Gibbs pdf(5) with $\mathrm{E}(\mathrm{H})$ using Gibbs sampling method [9].

4) Make update the fused image by using (10).

5) Reduce the looping variable and repeating the (2 and 3) steps till convergence.

6) Finding the upper and lower band intensity values from the resultant values.

\section{EXPERIMENTAL RESULTS}

As an example we have taken three multispectral images to demonstrate our algorithm and in evaluate the fusion scheme. The fig. 1 shows noisy source images, we improving quality of source images by using median filter and the enhanced images or as shown in fig. 2 the different bands multispectral source images. Each image is split or decompose into four sub images by using desecrate wavelet transform method in case of windowing scheme and fusion scheme approach is employed to contribute or fuse the images and finally we have to take the inverse wavelet transform for resultant image. In fig. 3 shows the fusion based results with our proposed algorithm and we present the performance resultant maximizing approach, LS approach, averaging approach, windowing based and our proposed approach. The maximizing approach is too bright resultant image. The LS approach is having drawback of showing more noise in resultant image and the resultant image produce by window based shows mosaic pattern.

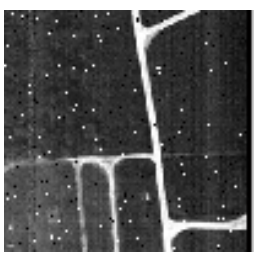

(a)

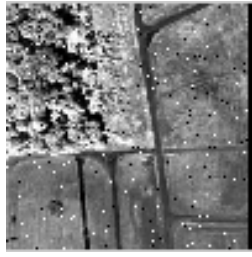

(b)

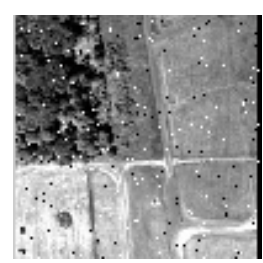

(c)

Fig. 1: Noisy source images
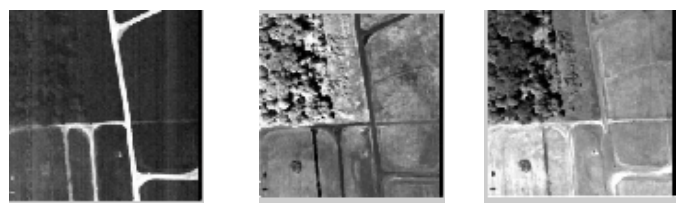

(a)

(b)

(c)

Fig.2: Enhanced source images

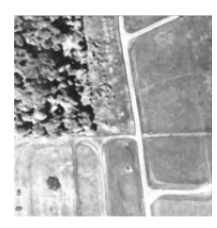

(a)

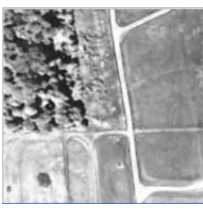

(d)

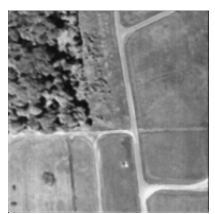

(b)

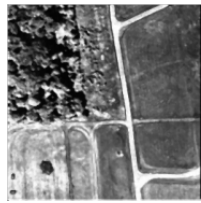

(e)

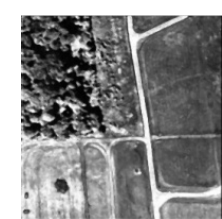

(c)

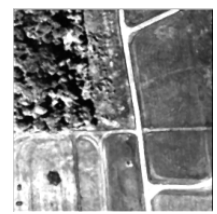

(f)
Fig.3: Fusion results based on (a) maximizing algorithm (b) averaging algorithm (c) LS algorithm (d) Window algorithm (e) MRF_H algorithm (f) Proposed algorithm.

\section{ACKNOWLEDGMENTS}

Here in this paper we have analyze the problem of image fusion on the basis of statistical model. We consider the importance of correlation and assume that it can also be modeled via MRF_H has been introduced. We presented different image fusion approaches such as LS, maximizing, averaging, windowing based and our proposed method. Which utilizes Gibbs pdf, Gibbs distribution and the results of which can be further more resultant fused image. Hear we assume a simple relation between true scene and input source image i.e. a source image may contribute or may not contribute fused image and we assume that the coefficients can take any real value in the data model. These may increase the accuracy of the image fusion.

\section{REFERENCES}

[1] Min Xu, "An image fusion approach based on markov random fields," IEEE Trans. Geosci. Remote Sens., vol. 49, No 12, dec. 2011.

[2] R. S. Blum, "On multi-sensor image fusion performance limits from an estimation theory perspective," Inf. Fusion, vol. 7, no. 3, pp. 250-263, Sep. 2006.

[3] Z. Wang, D. Ziou, C. Armenakis, D. Li, and Q. Li, "A comparative analysis of image fusion methods," IEEE Trans. Geosci. Remote Sens., vol. 43, no. 6, pp. 13911402, Jun. 2005

[4] C. Thomas, T. Ranchin, L. Wald, and J. Chanussot, "Synthesis of multispectral images to high spatial resolution: A critical review of fusion methods based on remote sensing physics," IEEE Trans. Geosci. Remote Sens., vol. 46, no. 5, pp. 1301-1312, May 2008.

[5] R. K. Sharma, T. K. Leen, and M. Pavel, "Probabilistic image sensor fusion," in Proc. Adv. Neural Inf. Process Syst. 11, 1999, pp. 824-830.

[6] P. Burt and R. Kolczynski, "Enhanced image capture through fusion," in Proc. 4th Int. Conf. Comput. Vis., 1993, pp.173-182. 
International Journal of Computer Applications Technology and Research

Volume *- No.*, 2012

[7] J. Yang and R. Blum, "A statistical signal processing approach to image fusion for concealed weapon detection," in Proc. IEEE Int. Conf. Image Process., 2002, pp. 513-516.

[8] E. Lallier and M. Farooq, "A real time pixel-level based image fusion via adaptive weight averaging," in Proc. 3rd Int. Conf. Inf. Fusion, 2000, pp. WEC3/3-WEC313.

[9] S. Z. Li, Markov Random Field Modeling in Computer Vision.New York: Spinger-Verlag, 2001.

[10] T. Kasetkasem and P. Varshney, "An image change detection algorithm based on Markov random field models," IEEE Trans. Geosci. Remote Sens., vol. 40, no. 8, pp. 1815-1823, Aug. 2002.

[11] Z. Tu and S. Zhu, "Image segmentation by data-driven Markov chain Monte Carlo," IEEE Trans. Pattern Anal. Mach. Intell., vol. 24, no. 5, pp. 657-673, May 2002.
[12] H. Chen, "Mutual information based image registration with applications," Ph.D. dissertation, Syracuse Univ., Syracuse, NY, May, 2002.

[13] S. Geman and D. Geman, "Stochastic relaxation, Gibbs distributions, and the Bayesian restoration of images," in Readings in Uncertain Reasoning. San Francisco, CA: Morgan Kaufmann, 1990, pp. 452-472.

[14] L. Bedini, A. Tonazzini, and S. Minutoli, "Unsupervised edge-preserving image restoration via a saddle point approximation," Image Vis. Comput., vol. 17, no.11, pp.779-793, Sep.1999.

[15] S. M. Kay, Fundamentals of Statistical Signal Processing: Estimation Theory. Upper Saddle River, NJ: Prentice-Hall, 1993.

[16] Y. C. Eldar, A. Beck, and M. Teboulle, "Bounded error estimation: A Chebyshev center approach," in Proc. 2nd IEEE Int. Workshop Compute. Adv. Multi-Sensor Adapt. Process, 2007, pp.205-208. 